\title{
SUMS AND PRODUCTS OF HILBERT SPACES
}

\author{
JESÚS M. F. CASTILLO
}

(Communicated by William J. Davis)

\begin{abstract}
Let $H$ be a Hilbert space. We prove that the locally convex sum $\bigoplus_{I} H$ is a subspace of the product $H^{J}$ if and only if $I$ is countable, $H$ is infinite dimensional, and card $J \geq 2^{\aleph_{0}}$.
\end{abstract}

Notations. For the general terminology on locally convex spaces we refer to [1, 2].

If $E$ is a locally convex space, $U(E)$ will denote a fundamental system of absolutely convex closed neighborhoods of 0 . If $p_{U}$ is the associated seminorm of $U \in U(E)$, we note $\widehat{E}_{U}$ for the completion of the normed space $\left(E / \operatorname{Ker} p_{U}\|\|_{U}\right)$, where $\left\|\phi_{U}(x)\right\|_{U}=p_{U}(x), \phi_{U}$ being the quotient map. The spaces $\widehat{E}_{U}$ will be referred as the associated Banach spaces. If $U, V \in U(E)$, $V \subset U$, the canonical linking map $\widehat{T}_{V U}$ is the extension to the completions of the operator $T_{V U} \in L\left(E_{V}, E_{U}\right)$ defined by $T_{V U} \phi_{V} x=\phi_{U} x$.

$K$ denotes the real or complex scalar field. If $I$ is a set of cardinality $d$ then $\varphi_{d}$ denotes the locally convex sum $\bigoplus_{I} K$ that is, the space $\bigoplus_{I} K$ endowed with the strongest locally convex topology. When $I=\mathbf{N}$ we simply write $\varphi$. Analogously, if $E$ is a locally convex space, $\bigoplus_{I} E$ denotes the sum space endowed with the strongest locally convex topology making all the inclusions $E \rightarrow \bigoplus_{I} E$ continuous.

Let $T$ be an operator acting between the Banach spaces $T: X \rightarrow Y$. Let $Z$ be a Banach space. By a subfactorization of $T$ through $Z$ we mean two operators $A: X \rightarrow Z$ and $B: \overline{\operatorname{Im}} A \rightarrow Y$ such that $T=B A$. Note that $B$ need not be defined on all of $Z$, but only on the closure of the range of $A$ in $Z$. When $B$ is defined on the whole $Z$ then we have a factorization of $T$ through $Z$.

The spaces $l_{p}(I), 0<p \leq+\infty$, are defined to be the Banach ( $p$-Banach if $0<p<1)$ spaces

$$
l_{p}(I)=\left\{\left(x_{i}\right)_{i \in I} \in K^{I}:\left\|\left(x_{i}\right)\right\|_{p}\right\}=\left\{\sum_{i \in I}\left|x_{i}\right|^{p}\right\}^{1 / p}<+\infty
$$

Received by the editors December 1, 1987 and, in revised form, March 22, 1988.

1980 Mathematics Subject Classification (1985 Revision). Primary 46B25, 46A12, 46A05, 46C10. 
if $p<+\infty$, and

$$
l_{\infty}(I)=\left\{\left(x_{i}\right)_{i \in I} \in K^{I}:\left\|\left(x_{i}\right)\right\|_{\infty}\right\}=\sup _{i \in I}\left|x_{i}\right|<+\infty
$$

if $p=+\infty$.

We write

$$
l_{\infty}^{+}(I)=\left\{x \in l_{\infty}(I): x_{i}>0 \forall_{i} \in I\right\}
$$

and recall the well-known fact that any Hilbert space is isometric with some $l_{2}(I)$.

Let $X$ be a Banach space, we will also consider the vector valued sequence spaces

and

$$
l_{p}(X)=\left\{\left(x_{n}\right) \in X^{N}:\left(\left\|x_{n}\right\|\right) \in l_{p}\right\}, \quad 1 \leq p<+\infty
$$

$$
c_{0}(X)=\left\{\left(x_{n}\right) \in X^{N}:\left(\left\|x_{n}\right\|\right) \in c_{0}\right\}
$$

which in fact are Banach spaces.

\section{MAIN RESULTS}

Theorem. Let $H$ be an infinite-dimensional Hilbert space. Then the locally convex sum $\bigoplus_{I} H$ is a subspace of some product $\prod_{J} H$ if and only if $I$ is countable and card $J \geq 2^{\aleph_{0}}$.

Obviously $H$ needs to be infinite-dimensional since $\varphi$, cannot be a subspace of any product $K^{J}$. On the other hand card $J \geq 2^{\aleph_{0}}$ is required since $\bigoplus_{N} H$ is not metrizable.

Proposition 1. $\bigoplus_{N} l_{2}$ is a subspace of $\prod_{J} l_{2}$, card $J \geq 2^{\aleph_{0}}$.

Proof. Since the locally convex sum topology coincides with the so-called boxtopology (see [1]) on countable sums, a fundamental system of neighborhoods of 0 is given by the sets: $U(z)=\prod_{N} z_{n} B \cap \bigoplus_{N} l_{2}$, where $B$ is the unit ball of $l_{2}$, and $z \in c_{0}$. We may suppose $z_{n} \neq 0$ for all $n \neq N$. Its associated seminorm is: $p_{z}\left(\left(x_{n}\right)\right)=\sup _{n} z_{n}^{-1}\left\|x_{n}\right\|_{2}$, and the associated Banach space is clearly seen to be the completion of $\bigoplus_{N} l_{2}$ endowed with the norm $p_{z}$, that is:

$$
\left\{\left(x_{n}\right) \in l_{2}^{N}: \sup _{n} z_{n}^{-1}\left\|x_{n}\right\|_{2} \rightarrow 0\right\} \text {. }
$$

This space is isometric with $c_{0}\left(l_{2}\right)$. Under this isometry, if $k \in c_{0}$ and $0<$ $k_{n} \leq z_{n}$, then the linking map between the associated Banach spaces to $p_{k}$ and $p_{z}$ is precisely the "diagonal" operator $D_{\sigma}: c_{0}\left(l_{2}\right) \rightarrow c_{0}\left(l_{2}\right), D_{\sigma}\left(\left(x_{n}\right)\right)=\left(\sigma_{n} x_{n}\right)$, where $\sigma_{n}=z_{n}^{-1} \mid k_{n}^{-1}$.

If we choose $k$ such that $\sigma$ belongs to $l_{2}$, then $D_{\sigma}$ factorizes through $l_{2}\left(l_{2}\right)$ :

$$
\begin{array}{cc}
c_{0}\left(l_{2}\right) & \stackrel{D_{\sigma}}{\longrightarrow} c_{0}\left(l_{2}\right) \\
& l_{\text {inclusion }} \\
& l_{2}\left(l_{2}\right)
\end{array}
$$




$$
\begin{aligned}
\left\|D_{\sigma} x\right\|_{l_{2}\left(l_{2}\right)} & =\left(\sum\left\|\sigma_{n} x_{n}\right\|_{2}^{2}\right)^{1 / 2}=\left(\sum\left|\sigma_{n}\right|^{2}\left\|x_{n}\right\|_{2}^{2}\right)^{1 / 2} \\
& \leq \sup _{n}\left\|x_{n}\right\|_{2}\left(\sum\left|\sigma_{n}\right|^{2}\right)^{1 / 2}=\|x\|_{c_{0}\left(l_{2}\right)}\|\sigma\|_{2} .
\end{aligned}
$$

The continuity of the inclusion is obvious. But the space $l_{2}\left(l_{2}\right)$ is isometric with $l_{2}$. Thus, the space $\bigoplus_{N} l_{2}$, as a projective limit of $l_{2}$ is a closed subspace of the topological product $\prod_{I} l_{2}[2,19.10 .3]$.

Remark. Since $l_{p}\left(l_{p}\right)$ is isometric with $l_{p}, 1 \leq p<+\infty$, the preceding proof serves for the spaces $l_{p}$, and with minor modications for the nonseparable spaces $l_{p}(I), 1 \leq p<+\infty$. Therefore it covers the situation for all Hilbert spaces.

Proposition 2. Let I be uncountable. Then $\bigoplus_{I} l_{2}$ is not a subspace of any product $\prod_{J} l_{2}$.

Proof. The latter space has separable associated Banach spaces while the former does not.

Proposition 3. Let $H$ be a Hilbert space, and I an uncountable set. Then $\bigoplus_{I} H$ is not a subspace of any product of copies of $H$.

Proof. We may write $H=l_{2}(I)$ with $I$ uncountable, by the remarks previous to Proposition 1, and Proposition 2. Let $I$ be uncountable with $d=\operatorname{card} I$.

Step 1. Let $A \in L\left(l_{2}(I), l_{1}(I)\right)$ represented by a matrix $\left(a_{i, j}\right)_{(i, j) \in I \times I}$ in the form:

$$
A\left(x_{j}\right)=\left(y_{i}\right) \text { with } y_{i}=\sum_{j \in I} a_{i j} x_{j} .
$$

Suppose that $A$ has (a) a row of zeros or (b) a column of zeros. Then $A$ cannot be part of a factorization of a diagonal operator $D_{\sigma}: l_{1}(I) \rightarrow l_{1}(I), \sigma \in l_{\infty}^{+}(I)$ through $l_{2}(I)$. In case (a) since then all the vectors in IM $A$ would have some coordinate zero, and $\operatorname{IM} A B \neq \operatorname{IM} D_{\sigma}$. In case (b) it is $A^{\prime}: l_{\infty}(I) \rightarrow l_{2}(I)$ which has a row of zeros and cannot be injective; since $D_{\sigma^{-1}}$ is injective, the factorization $D_{\sigma^{-1}}=D_{\sigma}^{\prime}=B^{\prime} A^{\prime}$ is impossible, and thus $D_{\sigma}=A B$ is impossible too.

From all this it follows that a nonzero element must exist in each row and in each column of $A$. Therefore the set $\left.\{i, j) \in I \times I: a_{i j} \neq 0\right\}$ is uncountable, and we may assume $a_{i j}>0$ for uncountable many pairs $(i, j)$. Thus an $\varepsilon>0$ must exist such that $a_{i j} \geq \varepsilon$ for an uncountable set $Z \subset I \times I$. Moreover these indexes of $Z$ need to be scattered through infinitely many rows and columns of $I \times I$; because if we suppose that they are "concentrated" in, let us say, a single column, then those vectors of $l_{2}(I)$ with the corresponding index zero have zero as the image by $A$. Since $B$ can be considered surjective (see Step 2 ), $A$ would not be a part of a factorization of $D_{\sigma}$. If they are "concentrated" on a row we obtain the same result by transposition. Therefore we can choose a countable set $Z_{0}=\left\{\left(i_{n}, j_{n}\right) \in Z, n \in N\right\}$ such that $i_{n} \neq i_{m}$ and $j_{n} \neq j_{m}$ 
whenever $n \neq m$. Choose then an element $\left(z_{j}\right) \in l_{2}(I) \backslash l_{1}(I)$ with $z_{j} \geq 0 \quad \forall j$ and $z_{j} \neq 0$ if some couple $(i, j) \in Z_{0}$. If $A z=y$, then we find that for each pair $(i, j) \in Z_{0}$ :

$$
y_{i}=\sum_{K \in I} a_{i k} z_{k} \geq \varepsilon z_{j}
$$

whence

$$
\sum_{i \in I}\left|y_{i}\right| \geq \varepsilon \sum_{j \in I}\left|z_{j}\right|=+\infty
$$

and $A$ cannot be an operator from $l_{2}(I)$ into $l_{1}(I)$. In this way we have essentially proved that:

Step 2. The diagonal operator $D_{\sigma}: l_{1}(I) \rightarrow l_{1}(I), \sigma \in l_{\infty}^{+}(I)$, cannot be subfactorized through $l_{2}(I)$ : the above manipulations settle the case of factorization. For subfactorizations we use orthogonal projection onto $\overline{\mathrm{IM} B}$ to obtain a factorization through a Hilbert space. If this is nonseparable the calculations of step 1 apply. If it is $l_{2}$ then $D_{\sigma}=A B, B \in L\left(l_{1}(I), l_{2}\right)$ and $A \in L\left(l_{2}, l_{1}(I)\right)$ is clearly false since the image of $D_{\sigma}$ cannot be contained in any $l_{1}(N)-N$ a countable subset of $I$.

Step 3. It is not hard to check that $\varphi_{d}$ has a fundamental system of neighborhoods of 0 with associated Banach spaces isometric with $l_{1}(I)$. Under this isometry the linking maps are the diagonal operators $D_{\sigma}, \sigma \in l_{\infty}^{+}(I)$.

Step 4. Let us assume that $\varphi_{d}$ is a subspace of some product $l_{2}(I)^{J}$. There is a fundamental system of neighborhoods of 0 in $l_{2}(I)^{J}, \mathscr{U}$, with associated Banach spaces isometric with $l_{2}(I)$. Thus an embedding of $\varphi_{d}$ into $l_{2}(I)^{J}$ would imply for $U \in \mathscr{U}$ the subfactorization

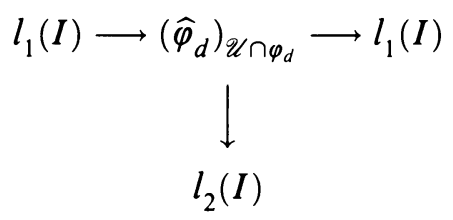

of $D_{\sigma}$, which we know is not possible.

Step 5. We complete the proof of our Proposition 3. Since the embedding of $\varphi_{d}$ into $H^{J}$ is not possible when $d$ is uncountable, the embedding of $\bigoplus_{I} H$ into $H^{J}$ is impossible too.

Remark. The result in Step 4 also holds when $\varphi_{d}$ and $l_{2}(J)$ with different index sets are considered. It is obviously true when card $J<d$ and, reasoning as in Step 2, when card $J>d$.

Remark. For general operators $T: l_{p}(I) \rightarrow l_{q}(J), I, J$ uncountable and $p>q$ we can obtain (compare with the final part of Step 1):

$$
\begin{aligned}
& \forall j \in I \quad \operatorname{card}\left\{i \in I: a_{i j} \neq 0\right\} \leq \aleph_{0}, \\
& \forall i \in I \operatorname{card}\left\{j \in I: a_{i j} \neq 0\right\} \leq \aleph_{0} \quad \text { if } p>1 .
\end{aligned}
$$


From this it follows that if $\operatorname{card}\left\{(i, j) \in I \times I: a_{i j} \neq 0\right\}>\aleph_{0}$ then these indexes cannot be "concentrated" in a countable number of rows or columns. We could then proceed as in Step 1 to obtain a contradiction. Therefore:

$$
\operatorname{card}\left\{(i, j) \in I \times I: a_{i j} \neq 0\right\} \leq \aleph_{0}
$$

and then $\operatorname{IM} T \subset l_{q}(N)$. We have

Lemma. Let $I, J$, be uncountable sets, $p>q \leq 1$ and $T: l_{p}(I) \rightarrow l_{q}(J) a$ continuous operator. Then $\operatorname{IM} T \subset l_{q}(N)$.

In the advance of this paper [4] the above lemma was incorreclty stated due to the omission of the hypothesis $p>1$. The next counterexample shows that in that way it is no longer true: consider a partition $I=\bigcup_{n=1}^{\infty} I_{n}$, with $I_{n}$ uncountable for all $n$. Take $I_{0}$ a countable subset of $I$, and define

$$
a_{i j}= \begin{cases}i^{-4} & \text { when } i \in I_{0} \text { and } j \in I_{i}, \\ 0 & \text { otherwise. }\end{cases}
$$

This matrix defines an operator from $l_{1}(I)$ to $l_{1 / 2}(I)$ for which $(*)$ does not hold.

\section{REFERENCES}

1. H. Jarchow, Locally convex spaces, Teubner, Stuttgart, 1981.

2. G. Kothe, Topological vector spaces. I, Springer-Verlag, 1969.

3. __ Topological vector spaces. II, Springer-Verlag, 1979.

4. Jesús M. F. Castillo, The sum problem for Hilbert spaces, Extracta Math. 3 (1988), 26-27.

Departamento de Matemáticas, Universidad de Extremadura, Avda de Elvas s/N, 06071-Badajoz, Spain 